\title{
Visualização dos dados abertos da Polícia Rodoviária Federal sobre acidentes nas rodovias brasileiras
}

\author{
Victor G. P. de Mattos, Pedro H. V. Vasconcelos, Yussef Parcianello, \\ Nádia P. Kozievitch, Rita Berardi \\ ${ }^{1}$ Universidade Tecnológica Federal do Paraná (UTFPR) \\ Avenida Sete de Setembro, 3165 \\ Departamento Acadêmico de Informática - DAINF - Curitiba - Brasil \\ \{victormattos, pedrovasconcelos\}ealunos.utfpr.edu.br, \\ yussef.parcianello@ifsc.edu.br, \{nadiap,ritaberardi\}@utfpr.edu.br
}

\begin{abstract}
Traffic accidents are one of the main causes of death in Brazil and, in the last 10 years, more than 1.6 million accidents along with 83 thousand deaths were registered. This paper proposes an open-source prototype for the visualization of open data provided by Polícia Rodoviária Federal (PRF), mapping historically the most dangerous sections of the highways, the most frequent causes of accidents, and helps to identify patterns. The purpose of this paper is to promote the awareness of users, aiming at preventing and reducing the number of accidents on Brazilian highways, which today, in addition to human loss, generate an average loss of $R \$ 10$ billion annually.
\end{abstract}

Resumo. Os acidentes de trânsito são uma das principais causas de óbito no Brasil e, somente nos últimos 10 anos, foram registrados mais de 1.6 milhões de acidentes e mais de 83 mil mortes. Este artigo propõe um protótipo de código aberto para a visualização dos dados abertos da Polícia Rodoviária Federal (PRF), mapeando os trechos historicamente mais perigosos nas rodovias, auxiliando na identificação de padrões. $O$ intuito deste artigo é promover a conscientização dos usuários, visando a prevenção e diminuição no número de acidentes nas rodovias brasileiras que, hoje, além da perda humana, geram um prejuizo médio de $R \$ 10$ bilhões anualmente.

\section{Introdução}

Cidade Inteligente é o termo dado à cidade que utiliza Tecnologias da Informação e Comunicação (TIC) de forma criativa, inclusiva e eficiente na gestão pública, auxiliando nas tomadas de decisão, bem como na criação de inovações que façam uso destas informações para gerar soluções que facilitem a vida dos cidadãos [Weiss et al. 2017].

Uma das peças-chave da gestão pública para a disseminação da informação e a promoção da transparência é a abertura de dados públicos, os chamados dados abertos. O aumento de dados públicos cria um cenário favorável à participação social, permitindo o cidadão ajudar a produzir informações que auxiliem na discussão e mapeamento de problemas, bem como o poder público na identificação dos mesmos [Lemos 2013].

Com o intuito de compreender melhor as características dos acidentes registrados pela PRF, o uso de tecnologias geoespaciais como os Sistemas de Informação Geográfica 
(SIG) proporciona, além de facilidade na análise, a criação de modelos espaciais que auxiliem no processo de tomada de decisões [Tao 2013].

A pesquisa de [Vila et al. 2016] propôs uma interface de visualização de dados geográficos com técnicas de clusterização utilizando a base de dados de mobilidade urbana utilizando técnicas de clusterização, permitindo o agrupamento de um grande conjunto de dados georreferenciados, tornando a visualização espacial mais intuitiva, como mostra a Figura 1 (esquerda).

Já o Instituto de Pesquisa e Planejamento Urbano de Curitiba (IPPUC) ${ }^{1}$ disponibiliza uma interface ${ }^{2}$ de visualização dos dados geográficos de acidentes de trânsito com vítimas fatais no município de Curitiba, como mostra a Figura 1 (direita). Além da visualização, o IPPUC também disponibiliza, em forma de gráficos, os dados mais relevantes destes acidentes, de modo que o usuário possa filtrar e definir quais dados devem aparecer nos gráficos.

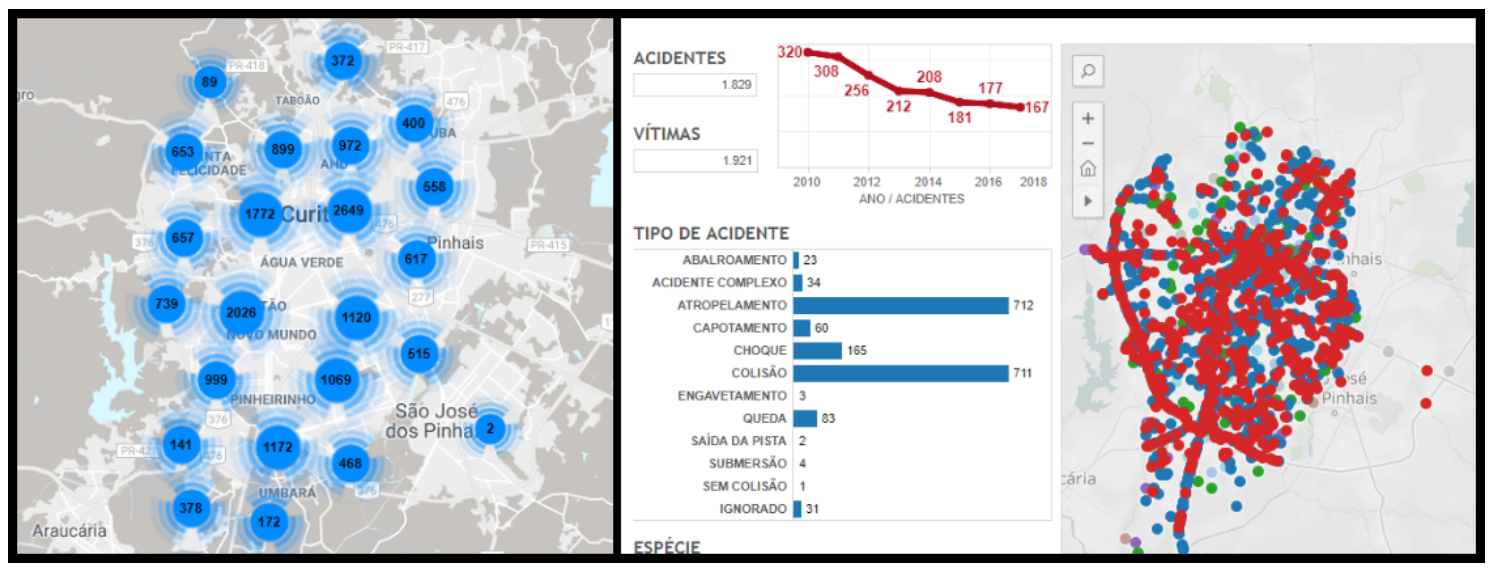

Figura 1. Agrupamento dos pontos de ônibus de Curitiba (esquerda) [Vila et al. 2016] e interface de visualização de acidentes de trânsito com vítimas fatais em Curitiba (direita) - IPPUC.

Em particular, dados de acidentes possuem não só componentes geográficos e temporais, como também dinâmicas incluindo trecho de BR, classificação diferenciada de acidentes, veículos e passageiros envolvidos em agregações diferenciadas (país, estado, cidade). Neste sentido, este trabalho em andamento utiliza como base os dois trabalhos acima para uma ferramenta visual de dados de acidentes, com o objetivo de suprir o problema de sobreposição de acidentes em áreas e identificação de padrões. Para isso, foram usados dados de uma década da $\mathrm{PRF}^{3}$.

O restante do trabalho está organizado da seguinte maneira: a seção 2 apresenta trabalhos relacionados. A seção 3 apresenta o protótipo da aplicação, e a seção 4 lista as conclusões e trabalhos futuros.

\footnotetext{
${ }^{1}$ www.ippuc.org.br - Acessado em: 09 de outubro de 2018.

${ }^{2}$ www.ippuc.org.br/mapasinterativos/acidentesDeTransito/dashboard.html - Acessado em: 17 de abril de 2019.

${ }^{3}$ https://www.prf.gov.br/portal/dados-abertos - Acessado em: 26 de junho de 2019.
} 


\section{Trabalhos Relacionados}

À medida que o mundo se torna cada vez mais instrumentado, interconectado e inteligente, as cidades têm a chance fazer uso de novas soluções e práticas de gerenciamento "inteligentes" [Dirks et al. 2009]. Com as cidades ficando mais inteligentes, uma grande quantidade de dados pode ser colocada à disposição da população e empresas, impulsionando o desenvolvimento de aplicações comerciais, pesquisas e apoio à tomada de decisões complexas [Lopes et al. 2016], contribuindo para o desenvolvimento de novos serviços de utilidade para cidadãos e gestores municipais [Cunha et al. 2016].

Neste contexto, os SIGs possuem como funcionalidades principais a captura, armazenamento, consulta, análise e exibição de dados geoespaciais [Chang 2015]. Longley [Longley et al. 2005] definem os SIG como uma extensão de sistemas de informação, que armazenam não só eventos e atividades, mas também onde estes acontecem. Para um acidente de trânsito em uma rodovia brasileira, como mostra [Kageyama et al. 2019], por exemplo, esses dados geoespaciais descrevem, além da localização, informações relacionadas como: em que rodovia ocorreu, em que quilômetro ocorreu, qual era o sentido da pista, o horário, a data, etc.

Com o aumento da quantidade de dados e informações disponíveis, torna-se cada vez mais necessário o uso de ferramentas e técnicas que ajudem a fazer uso efetivo desse excesso de informação, como por exemplo, a visualização de dados. Dentro do contexto deste projeto, das aplicações de visualização de dados de acidentes de trânsito pesquisadas, destacam-se: o New York City Open Data ${ }^{4}$ (Figura 2 - canto superior esquerdo), o Mapping 10 Years of Fatal Traffic Accidents ${ }^{5}$ (Figura 2 - canto superior direito), o The Boston Crash Model ${ }^{6}$ (Figura 2 - canto inferior esquerdo) e o Seattle Collisions ${ }^{7}$ (Figura 2 - canto inferior direito).

Todas as aplicações citadas utilizam o OpenStreetMap e, com exceção do Seattle Collisions, que faz uso de bibliotecas do Javascript, são utilizadas ferramentas pagas especializadas em visualização de mapas. Além disso, a maioria delas não faz uso de ferramentas de clusterização ou visualização (o que gera sobreposição dos dados e dificulta o entendimento dos mesmos), além de não possuirem código aberto. Este trabalho propõe o uso de ferramentas de clusterização para a visualização no intuito de facilitar a identificação de padrões.

\section{Protótipo}

Aproximadamente 10 anos de dados de acidentes de todo o Brasil foram inseridos em um servidor PostGIS [Obe and Hsu 2011], com aproximadamente 1 milhão de tuplas, entre os anos de 2007 e 2018. Detalhes dos dados (como o menor número de acidentes ao longo dos últimos 5 anos, a concentração de mortes em fins de semana, e a preferência dos acidentes pelo horário das 18 horas e pelo mês de dezembro, entre outros) podem ser verificados em [Kageyama et al. 2019].

O protótipo de visualização foi desenvolvido para a web e utiliza a arquitetura apresentada na Figura 3: PostgreSQL (11.0), PostGIS (6.5.2) (camada de dados), Node.js

\footnotetext{
${ }^{4}$ www.opendata.cityofnewyork.us - Acessado em: 18 de setembro de 2018.

${ }^{5}$ www.metrocosm.com/10-years-of-traffic-accidents-mapped.html - Acessado em: 10 de abril de 2018.

${ }^{6}$ www.apps.boston.gov/vision-zero - Acessado em: 15 de outubro de 2018.

${ }^{7}$ www.seattlecollisions.timganter.io/collisions - Acessado em: 15 de outubro de 2018.
} 


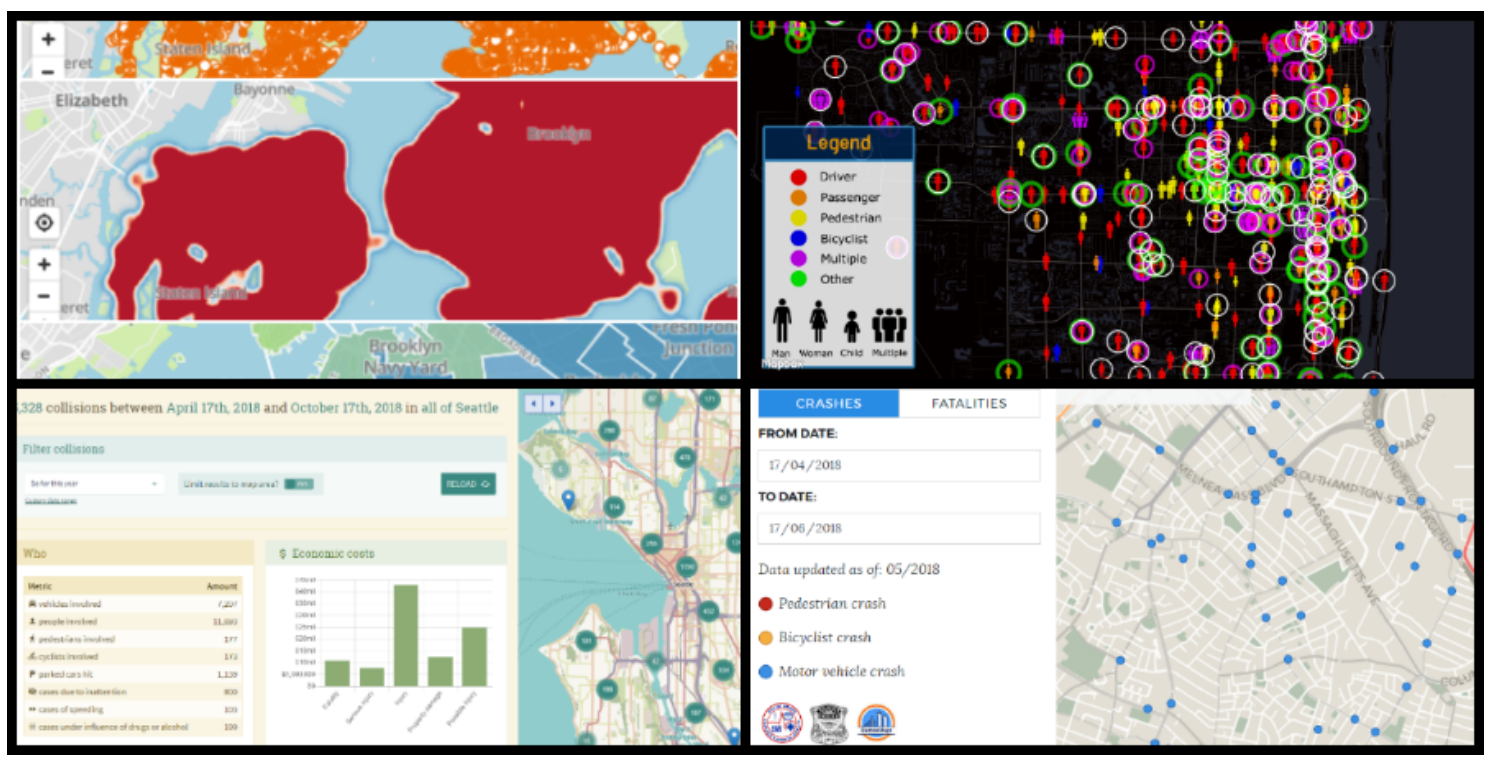

Figura 2. Aplicações de visualização de acidentes de trânsito.

(8.12.0) e Express (4.17.0) (camada de processamento) e HTML, CSS, JavaScript, D3 (3.5.17) e Leaflet (0.7.2) (camada de apresentação).

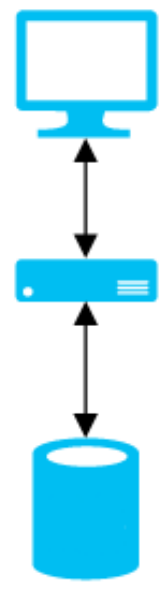

Camada de apresentação
Tecnologias: HTML, CSS, JavaScript, D3 e Leaflet

\section{Camada de processamento}

Tecnologias: Node.js e Express

\section{Camada de dados}

Tecnologias: PostgreSQL e PostGIS

\section{Figura 3. Arquitetura da aplicação.}

A interface de resultado foi inspirada em interfaces já utilizadas pelo governo local (IPPUC), e está dividida entre o mapa que contém os acidentes (mostrado na Figura 4) e em outra metade com as estatísticas gerais dos acidentes (mostrado na Figura 5). Os gráficos podem ser reproduzidos através de filtros como: dia da semana, ano, horário, região e tipo de acidente.

$\mathrm{Na}$ Figura 4, os acidentes estão agrupados em clusters que representam a quantidade de acidentes em determinada rodovia. Além disso, os clusters foram classificados, inicialmente, em acidentes com vítimas fatais, vítimas feridas e sem vítimas, cada um com uma cor específica descrita no índice ao lado do mapa (parte superior da Figura 4). A clusterização no mapa é ajustada dinamicamente de acordo com a posição e zoom, 
como na parte inferior da Figura 4, que mostra o zoom na cidade de Curitiba e sua região metropolitana, evidenciando as rodovias em que ocorreram os acidentes e a reorganização dos clusters (opções não disponíveis em ferramentas locais do governo como IPPUC, por exemplo).

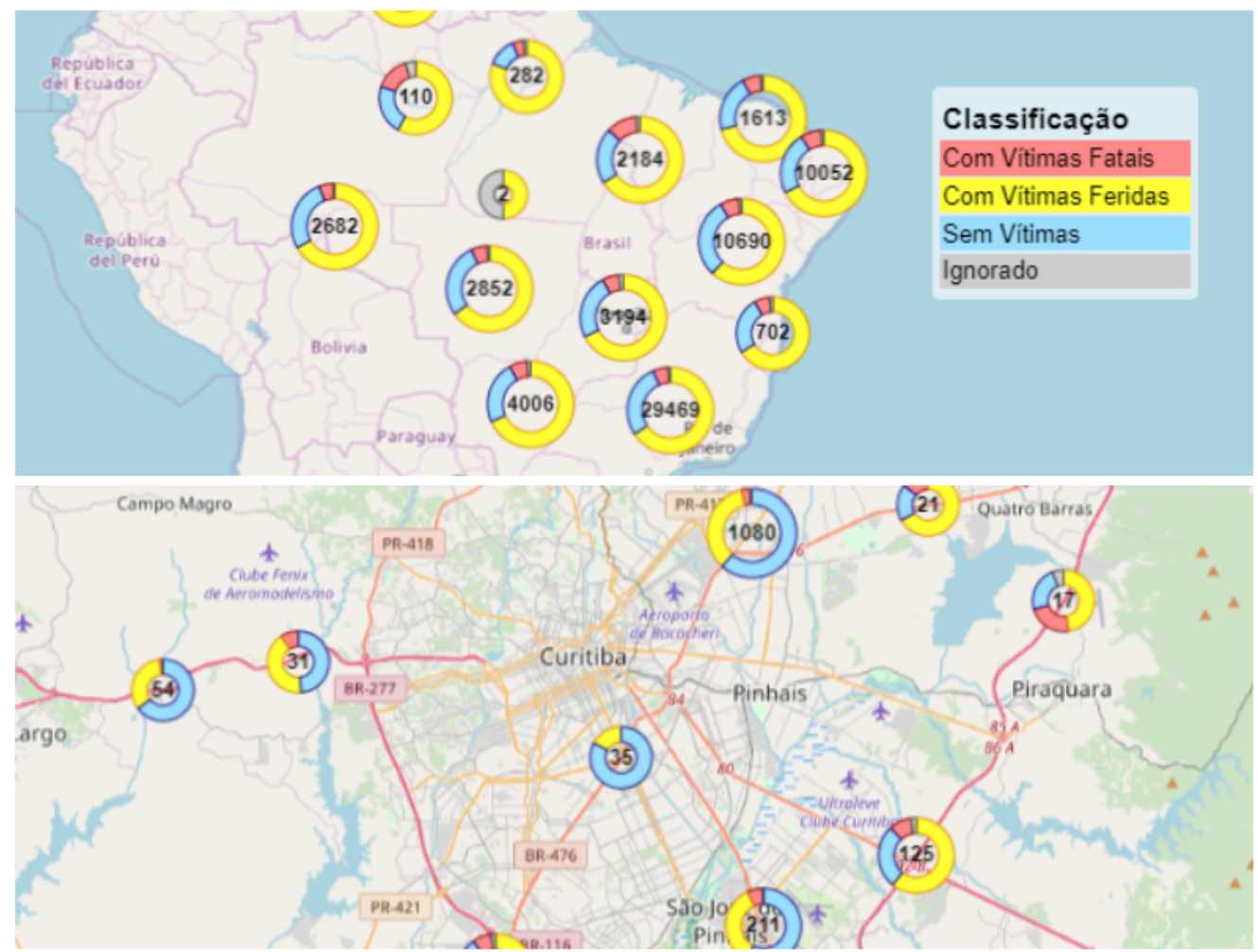

Figura 4. Protótipo da aplicação.

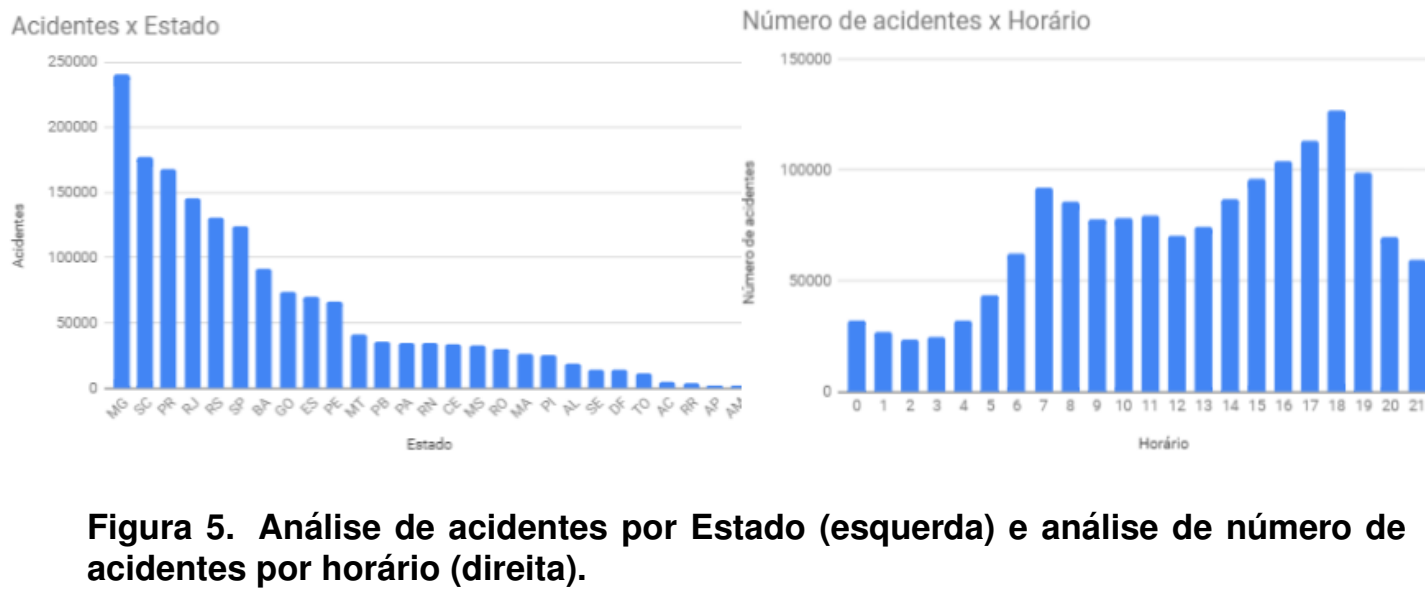

Além do protótipo, um questionário para validar requisitos ${ }^{8}$ foi desenvolvido para ser submetido a PRF e ao público em geral. O questionário possui perguntas básicas como: "Você faz uso de alguma rodovia federal?", "Qual a frequência com que você utiliza as rodovias federais?", "Você, algum conhecido ou familiar, já se envolveu em

\footnotetext{
${ }^{8}$ https://forms.gle/7fhcWQF57aDrq24u9 - Acessado em: 09 de julho de 2019.
} 
algum acidente de trânsito nas rodovias brasileiras?", etc. Após a finalização do projeto, outro questionário será realizado para validar a ferramenta.

\section{Conclusão}

Este artigo apresentou um trabalho em andamento de uma ferramenta visual de dados de acidentes, com o objetivo de suprir o problema de sobreposição de dados em áreas e identificação de padrões. Através da utilização do protótipo, é possível tomar ciência da quantidade de acidentes de trânsito nas rodovias brasileiras, assim como é possível também identificar onde tais acidentes ocorrem, o que permite identificar as regiões mais violentas em termos de acidentes com vítimas fatais.

Como trabalho futuro, a este trabalho poderia ser adicionado os dados de redutores de velocidade, veículos e pessoas envolvidos em acidentes, além de outras estatísticas por período, como meses com maior número de acidentes, localização onde os mesmos ocorrem, as principais causas, e períodos com festas.

\section{Referências}

Chang, K.-T. (2015). Introduction to geographic information systems. McGraw-Hill Higher Education Boston, Boston.

Cunha, M. A., Przeybilovicz, E., Macaya, J. F. M., and Santos, F. B. P. d. (2016). Smart cities: Transformação digital de cidades.

Dirks, S., Keeling, M., and Dencik, J. (2009). How smart is your city?: Helping cities measure progress. IBM Institute for Business Value, IBM Global Business Services, New York.

Kageyama, M., Kozievitch, N. P., and Berardi, R. (2019). Acidentes nas rodovias brasileiras nos ultimos 10 anos: uma análise com dados abertos. In XV Escola Regional de Banco de Dados, pages 79-87.

Lemos, A. (2013). Cidades inteligentes. GV-executivo, 12(2):46-49.

Longley, P. A., Goodchild, M. F., Maguire, D. J., and Rhind, D. W. (2005). Geographic information systems and science. John Wiley \& Sons, England.

Lopes, G., Vidal, V. M. P., and Oliveira, M. (2016). Construção de linked data mashup para integração de dados da saúde pública. In SBBD 2016, pages 145-150.

Obe, R. and Hsu, L. (2011). Postgis in action. GEOInformatics, 14(8):30.

Tao, W. (2013). Interdisciplinary urban gis for smart cities: advancements and opportunities. Geo-spatial Information Science, 16(1):25-34.

Vila, J., Kozievitch, N. P., Gadda, T. M. C., Fonseca, K., Rosa, M. O., Gomes, L. C., and Akbar, M. (2016). Urban mobility challenges - an exploratory analysis of public transportation data in curitiba. Revista de Informática Aplicada, 12(1).

Weiss, M. C., Bernardes, R. C., and Consoni, F. L. (2017). Cidades inteligentes: casos e perspectivas para as cidades brasileiras. Revista Tecnológica da Fatec Americana, 5(1):01-13. 